\title{
The advancement of immunotherapy in hepatocellular carcinoma
}

\author{
Ling $\mathrm{Ni}^{1}$, Yu Feng ${ }^{1}$, Chen Dong ${ }^{1,2}$ \\ IInstitute for Immunology and School of Medicine, Tsinghua University, Beijing 100084, China. \\ ${ }^{2}$ Beijing Key Lab for Immunological Research on Chronic Diseases, Beijing 100084, China.
}

Correspondence to: Dr. Chen Dong, Institute for Immunology and School of Medicine, Tsinghua University, Medical Research Building, No.30 Haidian Shuangqing Road, Beijing 100084, China. E-mail: chendong@tsinghua.edu.cn

How to cite this article: Ni L, Feng Y, Dong C. The advancement of immunotherapy in hepatocellular carcinoma. Hepatoma Res 2020;6:25. http://dx.doi.org/10.20517/2394-5079.2020.14

Received: 12 Feb 2020 First Decision: 16 Mar 2020 Revised: 2 Apr 2020 Accepted: 10 Apr 2020 Published: 11 May 2020

Science Editors: Jia Fan, Ying-Hong Shi Copy Editor: Jing-Wen Zhang Production Editor: Jing Yu

\begin{abstract}
Most patients diagnosed with hepatocellular carcinoma (HCC) present with advanced or metastatic disease. The lack of therapeutic options in the treatment of advanced HCC accounts for its high mortality and recurrence rate. HCC is known as an immunogenic tumor, which develops in chronically inflamed livers. Anti-PD-1/PD-L1 antibodies (immune checkpoint inhibitors, ICB) were approved by the FDA to treat advanced HCC in patients previously treated with sorafenib as a second line. This has opened up a new era of anticancer treatment, although the response rate of HCC to anti-PD-1/PD-L1 antibodies is only around 20\%. Other than ICB treatment, adoptive cell transfer, dendritic cell-based vaccines and oncolytic therapy are currently under clinical trials. In this review, different immunotherapy approaches for HCC is presented. Current knowledge on the mechanisms of action for each approach is discussed and relevant, ongoing clinical trials are presented. We also discuss the future of immunotherapy and combination treatment for HCC patients.
\end{abstract}

Keywords: Hepatocellular carcinoma, immunotherapy, anti-PD-1/PD-L1 antibodies

\section{INTRODUCTION}

Hepatocellular carcinoma (HCC) is the most common primary liver cancer, and the second most common cause of cancer-related death worldwide. The incidence of HCC is higher in China and may account for $50 \%$ of new cases globally each year ${ }^{[1]}$. Most HCCs in China and South-East Asia are caused by the hepatitis

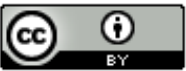

(C) The Author(s) 2020. Open Access This article is licensed under a Creative Commons Attribution 4.0 International License (https://creativecommons.org/licenses/by/4.0/), which permits unrestricted use, sharing, adaptation, distribution and reproduction in any medium or format, for any purpose, even commercially, as long as you give appropriate credit to the original author(s) and the source, provide a link to the Creative Commons license, and indicate if changes were made.

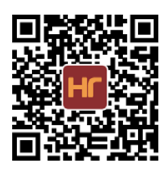


$B$ virus while in the West, nonalcoholic-associated steatosis seems to be one of the main causes ${ }^{[2]}$. In the early stages of HCC, hepatic resection or liver transplantation is first-line treatment with a high probability of a recurrence-free postoperative course. Sorafenib, a broad tyrosine kinase inhibitor, is standard treatment for advanced or metastatic HCC ${ }^{[3]}$. HCC is also known as an immunogenic tumor, which develops in chronically inflamed livers from both viral and non-viral causes. This inflammation promotes tumor development and is related with increased tumor immunogenicity ${ }^{[4]}$. As such, immunotherapy might be a more appropriate treatment strategy for HCC.

The liver, however, plays a pivotal role in host defense and the maintenance of immune competence. It is known to have an intrinsic,immunosuppressive microenvironment, which may serve as a major barrier to cancer immunotherapy ${ }^{[4]}$. Moreover, the low expression levels of tumor-associated antigens result in weaker $\mathrm{T}$ cell activation and in turn, tumor infiltration, which results in less efficient tumor control and consequently, a poorer clinical outcome. Thus, therapeutic strategies need to be developed against the HCC tumor suppressive microenvironment, which is involved in lowering the efficacy of immunotherapy ${ }^{[5]}$.

Although treatment strategies for patients with intermediate and advanced HCC are quite limited, an attractive option is immunomodulation therapy ${ }^{[6]}$. Current HCC immunotherapy approaches include immune checkpoint inhibitor antibodies (ICB), adoptive cell transfer (ACT), dendritic cell-based vaccines and oncolytic therapy but only ICB (anti-PD-1/PD-L1 antibodies) have been approved by the FDA as second-line treatment for HCC patients ${ }^{[7]}$. The major impediment to the development and success of immunotherapy in HCC patients is the intrinsic immunosuppressive microenvironment ${ }^{[6]}$. In this review article, we summarize recent advances in, and the future of immunotherapy for HCC and discuss their combination for the treatment of HCC patients.

\section{TUMOR MICROENVIRONMENT}

The tumor microenvironment (TME) is widely studied for the identification of novel therapeutic targets. The HCC TME consists of cancer and stromal cells, including hepatic stellate cells, cancer-associated fibroblasts, immune cells and endothelial cells, which interact with and affect the growth of the tumor ${ }^{[8]}$. The immune landscape of HCC is heterogeneous. By using 10x-single cell RNA-sequencing, Zhang and colleagues found that intratumor $\mathrm{LAMP}^{+}$mature dendritic cells (DCs) had the capacity to migrate from tumors to lymph nodes ${ }^{[9]}$. LAMP $^{+}{ }^{+}$DCs also expressed diverse immune-relevant ligands and possessed the ability to regulate several types of lymphocytes. Macrophages in tumors displayed distinct transcriptional states and tumor-associated macrophages (TAMs) were correlated with a poor prognosis. Myeloid cells in ascites were predominantly of tumor origin while lymphoid cells originated from blood ${ }^{[9]}$. Through time-of-flight mass cytometry, Chew et al. ${ }^{[10]}$ found that the HCC TME was enriched in regulatory T cells, tissue resident memory $\mathrm{CD}^{+} \mathrm{T}$ cells, resident natural killer cells, and TAMs. Tissue resident memory $\mathrm{PD}-1^{+} \mathrm{CD} 8^{+} \mathrm{T}$ cells were the major $\mathrm{T}$ cell subset responsive to PD-1 blockade. Zhang et al ${ }^{[11]}$ found that B cell infiltration of tumors was significantly impaired during tumor progression but tumors with a high density of B cell infiltration correlated with better clinical outcomes. It was also found that there was a high number of TIM-1 ${ }^{+}$Breg cell infiltrating the tumors of HCC patients ${ }^{[12,13]}$. These infiltrating Breg cells expressed IL-10 and had high suppressive activity against $\mathrm{CD} 8^{+}$and $\mathrm{CD} 4^{+} \mathrm{T}$ cells. B cells, except Breg cells, may contribute to tumor regression. Thus, using a selective reagent to inhibit suppressive B cells may be a viable strategy. By improving understanding of the immunologic components of the TME and its corresponding interactions at the biological and molecular levels, more effective immunotherapies for HCC can be designed.

\section{ADVANCEMENT OF ICB}

The mechanism of action of ICB is to stimulate or normalize the immune system of cancer patients by targeting negative regulators of $\mathrm{T}$ cell signaling pathways. FDA approved PD-1/PD-L1 inhibitor antibodies 
can be used to treat advanced HCC in patients previously on sorafenib as second line treatment ${ }^{[7]}$. HCC however, shows relatively low responsiveness to PD-1/PD-L1 inhibitor antibodies with an objective response rate of only around $20 \%{ }^{[14]}$.

Factors regulating and predictive of the response to anti-PD-1/PD-L1 immunotherapy in HCC are still largely unknown. To test whether PD-L1 expression on tumor cells can serve as a biomarker for PD-1 blockade therapy, Ou et al. ${ }^{[15]}$ used a mouse liver cancer cell line BNL-MEA transfected with a PD-L1 plasmid to establish an orthotopic HCC model. Wild-type (WT) and PD-L1-expressing tumor-bearing mice were then treated with PD-1 inhibitor antibodies. PD-L1-expressing tumors showed better response to anti-PD- 1 therapy than WT tumors. In addition, depletion of $\mathrm{CD}^{+} \mathrm{T}$ cells abolished the anti-tumor responses. Thus, PD-L1 expression in HCC cells may contribute to tumor immune evasion by suppressing $\mathrm{T}$ cell function. In turn, PD-L1 expression on tumor cells can be a biomarker for PD-1 ICB in this rodent model of HCC. However, the use of PD-L1 expression on human HCC cells as a biomarker needs to be investigated further. In a study of HCC patients treated with anti-PD-1 antibodies, the responders' (responding to PD-1 ICB) fecal samples exhibited higher taxa richness and more gene counts compared to those from non-responders. During PD-1 inhibitor immunotherapy, dynamic analysis showed that the dissimilarity of beta diversity displayed was obvious between patients from as early as Week 6 . Proteobacteria increased from week 3 and became predominant at week 12 in non-responders. The dynamic variation in characteristics of the gut microbiome may predict immunotherapy outcomes in HCC, which is important for disease-monitoring and treatment decision-making ${ }^{[16]}$.

Due to the low response rate to anti-PD-1 monotherapy, combining immunotherapy has become a new direction for future exploration in HCC treatment. Combination therapy of PD-1 ICB with targeted therapy, CTLA-4 ICB, oncolytic immunotherapy, epigenetic modulation and radiotherapy are all worthy of attention.

Recently, anti-PD-L1 therapy in combination with anti-VEGF antibodies demonstrated success based on the Phase III IMbrave150 trial in 501 patients with unresectable HCC who had not received previous systemic therapy. Participants were split 2:1 to receive the combination of anti-PD-L1 and anti-VEGF antibody versus sorafenib. Both anti-PD-L1 and anti-VEGF were administered intravenously whereas sorafenib is an oral medication. Patients in both arms received treatment until unacceptable toxicity developed or when there was no longer any clinical benefit. The trial met its co-primary endpoints, showing statistically significant and clinically meaningful improvements in overall survival (OS) and progression-free survival compared to the standard-of-care, sorafenib. Safety were consistent with those known for both anti-PD-L1 and anti-VEGF antibodies (ClinicalTrials.gov Identifier: NCT03434379). These findings indicate that the PD-L1/VEGF inhibitor combination can improve the efficacy of ICB.

The combined immunotherapy of anti-PD-1 with anti-CTLA-4 is now in a phase II trial on treating patients with resectable HCC (ClinicalTrials.gov Identifier: NCT03222076). Recently a case that achieved complete response with such combined therapy was reported. There was correlation of clinical response with increased $\mathrm{CD}^{+} \mathrm{T}$ cell infiltration, as well as increased effector $\mathrm{T}$ cell clusters. The study is ongoing and final results may contribute to a paradigm shift in the perioperative treatment of $\mathrm{HCC}^{[17]}$.

Enhancer of Zeste Homolog 2 (EZH2) and DNA Methyltransferase 1 (DNMT1) are two components of epigenetic modulation. In a mouse HCC model, combining anti-PD-L1 therapy with either DZNep (EZH2 inhibitor) or 5-Azacytidine (DNMT1 inhibitor) resulted in significantly increased tumor regression compared to epigenetic therapy or immunotherapy alone, which suggests that epigenetic modulation might be a potential, novel strategy to enhance immunotherapy for $\mathrm{HCC}^{[18]}$. Mechanistically, epigenetic modulators can activate transcriptionally repressed chemokine genes and stimulate $\mathrm{T}$ cell trafficking into 
the TME. In addition, these modulators can also induce previously silent neoantigens and elicit neoantigenspecific $\mathrm{T}$ cell responses to control tumors ${ }^{[18]}$.

Combination therapy with yttrium-90 radioembolization and PD-1 ICB for HCC treatment was also studied $^{[19]} .26$ consecutive HCC patients who received PD-1 ICB and radioembolization from April 2015 to May 2018 were included in a single-center, retrospective study. The results indicate that PD-1 ICB combined with yttrium-90 radioembolization in cases of HCC appears to be well-tolerated. Future prospective studies are needed to optimize treatment protocols and evaluate the efficacy of combination therapy ${ }^{[19]}$.

\section{ADVANCEMENT OF DENDRITIC CELL-BASED HCC VACCINES}

The response of HCC to ICB has been disappointing and new strategies are being explored. Tumorassociated antigen-loaded DCs are a potential immunotherapy for cancer, since DCs are the strongest professional antigen-presenting cells, which can orchestrate the innate and adaptive immune systems. Sipuleucel-T, the first FDA-approved DC vaccine, was observed to be effective in the treatment of human prostate cancer to some extent ${ }^{[20]}$. In the context of HCC, DC-based immunotherapy has recently been reported. Although HCC in China develops from chronic hepatitis B virus (HBV) infection, most HCC cells containing HBV-DNA fragments do not encode entire HBV antigens, but HBV epitopes can be encoded. To date, several tumor-associated antigens have been identified for DC-based vaccines for the treatment of patients with HCC including alpha-fetoprotein, glypican-3, melanoma associated antigen-1 and aspartate $\beta$-hydroxylase.

Alpha-fetoprotein (AFP) is a glycoprotein derived from embryonic endoderm tissue. Expression levels of AFP in fetal serum are high and decrease over time. While mature hepatocytes lose the ability to synthesize AFP, liver cancer cells can synthesize AFP after transformation. Hence, AFP has been detected in several malignant tumors, including that of the stomach, pancreas and liver. The majority of human HCC cells express high levels of AFP in Eastern populations ${ }^{[21]}$. Therefore, HCC patients with negative or weakly positive AFP in serum are associated with highly differentiated cancers. Glypican-3 (GPC3), another tumor-associated antigen, is a heparan sulfate proteoglycan, which binds to glycosylphosphatidylinositolanchored proteins and can be detected in the liver and kidneys of healthy fetuses. Adults express low or no GPC3, except in the placenta. GPC3 is also specifically expressed in several types of cancers including HCC, ovarian clear cell carcinoma, melanoma, squamous cell carcinoma of the lung, hepatoblastoma, nephroblastoma (Wilms tumor), yolk sac tumors, and some pediatric cancers. In some trials, HLA-A24and A2-restricted GPC3-derived peptide vaccines elicited GPC3-specific cytotoxic T cells in most vaccinated patients and in turn, improved outcomes ${ }^{[22]}$. Melanoma antigen 1 family contains a lot of chromosome X-clustered genes, including MAGE-1, MAGE-B and MAGE-C groups. Most of them cannot be detected in normal adult tissues except in the testis ${ }^{[23]}$. However, various types of cancers highly express it, including HCC. MAGE-1 protein-derived peptides are currently being studied as targets for the development of cancer vaccines ${ }^{[24]}$. Aspartate $\beta$-hydroxylase (ASPH), a type II transmembrane protein, is a highly conserved dioxygenase enzyme, which is overexpressed in a variety of cancers, including HCC. Normal adult tissues rarely express ASPH, except in placental trophoblastic cells. In HCC patients, ASPH overexpression was significantly correlated with higher relapse and lower survival rates after surgery, and could also predict worse surgical outcomes in early-stage HCC patients ${ }^{[25]}$. The expression pattern of ASPH makes it a potential biomarker and therapeutic target in cancer.

In phase I/IIA ${ }^{[26]}$ and phase $\mathrm{II}^{[27]}$ trials, Lee et al ${ }^{[26,27]}$ showed that autologous DC vaccines prepared by loading with the three most common HCC TAAs (AFP, MAGE-1, and GPC-3) in order to cover HCC heterogeneity were safe and well tolerated in HCC patients. In addition, DC vaccination led to enhanced tumor-specific immune responses. Therefore, adjuvant immunotherapy with DC vaccines decreases the 
risk of tumor relapse in HCC patients who have undergone standard treatment.

ASPH was also utilized to make DC-based HCC vaccines. An adenovirus vector encoding the ASPH gene (Ad-ASPH-IRES2-EGFP) was constructed and bone marrow-derived DCs were infected by Ad-AAHIRES2-EGFP to prepare ASPH-DC vaccines. After infection, DCs showed a mature phenotype with higher expression of CD11c, CD80, and MHC-II. Co-culture of ASPH-DCs and T cells resulted in enhanced killing capacity of T lymphocytes on the HepG2 HCC cell line. In addition, this ASPH-DC vaccine also improved the killing function of cytotoxic T lymphocytes (CTLs) compared to controls in an animal model. Therefore, this finding indicates that the ASPH-DC vaccine may be a potential candidate for DCbased immunotherapy of $\mathrm{HCC}^{[28]}$. However, for the ASPH-DC vaccine, only one molecule, ASPH, can be targeted, which cannot account fully for HCC heterogeneity.

From these studies, Chen et al. ${ }^{[29]}$ conducted a systematic review and meta-analysis to evaluate the clinical efficacy of DC-based vaccines in treating HCC. In total, there were 1276 cases from 19 clinical trials. They found that DC-based vaccines not only improved tumor control and increased the survival rate of HCC patients, its toxicity was also well-tolerated. These findings will provide new insight towards further development of DC-based vaccines as an adjuvant treatment strategy. However, the sample size, publication biases, varied study designs, pre-treatment and therapeutic processes of DCs have to be taken into consideration when evaluating DC-based immunotherapy.

\section{ADVANCEMENT OF ACT}

Cellular immunotherapy appears to be a promising modality for the treatment of malignant tumors. The mechanism of action in adoptive cell transfer is to treat cancer patients with their own naturally occurring or genetically modified anti-tumor lymphocytes to control tumors. Several meta-analyses have confirmed the evidence that adjuvant ACT for HCC patients after curative treatment reduces the risk of mortality and tumor relapse $\mathrm{s}^{[30,31]}$.

\section{Chimeric antigen receptor $\mathrm{T}$ cells}

Chimeric antigen receptor $\mathrm{T}$ cells (CAR-T cells) are $\mathrm{T}$ cells that have been engineered to express an artificial $\mathrm{T}$ cell receptor. The FDA has approved CAR-T cells that target CD19 to treat myeloma, a hematological malignancy derived from plasma cells ${ }^{[32]}$. However, the use of CAR-T cells to treat solid tumors is limited due to the scarcity of tumor-specific antigen targets and the poor infiltration of CAR-T cells into tumor tissue. In the context of HCC, CAR-T cells are still under investigation. Currently, these targets include GPC-3, CD133, NKG2D and CD147.

GPC3-targeted CAR-T cells could be a promising therapeutic option for $\mathrm{HCC}^{[33-35]}$. Gao et al. ${ }^{[33]}$ found that GPC3-CAR-T cells could efficiently eliminate GPC3 positive HCC cells but not GPC3 negative cells in vitro, and their cytotoxic activities might be positively associated with the expression levels of GPC3 on target cells. Moreover, third-generation GPC3-CAR-T cells could eliminate HCC xenografts with high GPC3 expression and potently impair HCC xenograft growth with low expression levels of GPC3 in vivo. Treatment with third-generation GPC3-targeted CAR-T cells also significantly prolonged the survival of mice bearing established orthotopic Huh-7 xenografts. In addition, in order to reduce on-target, off-tumor toxicity by GPC3-CAR-T cells, CAR-T cells targeting GPC3 and asialoglycoprotein receptor 1 (ASGR1) (a liver tissue-specific protein) were prepared. Dual-targeted CAR-T cells carry anti-GPC3-CD3 $\zeta$ for primary signal transduction and anti-ASGR1-28BB for co-stimulatory signal transduction. The results showed that dual-targeted CAR-T cells had no cytotoxic effect on $\mathrm{ASGR}^{+} \mathrm{GPC} 3^{-}$tumor cells but killed $\mathrm{GPC} 3^{+} \mathrm{ASGR} 1$ and $\mathrm{GPC} 3^{+} \mathrm{ASGR} 1^{+} \mathrm{HCC}$ cells in vitro. Moreover, the dual-targeted CAR-T cells potently suppressed the growth of GPC $3^{+} \mathrm{ASGR} 1^{+} \mathrm{HCC}$ tumor xenografts while no obvious growth inhibition was seen with either single or double antigen-negative tumor xenografts. Additionally, the dual-targeted T cells showed higher 
anti-tumor capacity and persistence than single-targeted T cells in two GPC ${ }^{+} \mathrm{ASGR}^{+}{ }^{+} \mathrm{HCC}$ xenograft models. Taken together, dual-targeted CAR-T cells may decrease the risk of on-target, off-tumor toxicity while maintaining relatively potent antitumor capacities in $\mathrm{GPC} 3^{+} \mathrm{ASGR} 1^{+} \mathrm{HCC}^{[36]}$.

The combination of CAR-T cell therapy and knockout of endogenous inhibitory immune checkpoints on $\mathrm{T}$ cells might be a promising immunotherapeutic approach for cancer treatment. The $P D-1$ gene in GPC3-targeted CAR-T cells was knocked out via the CRISPR/Cas9 gene-editing system. In vitro, $\mathrm{PD}-{ }^{-1}{ }^{-}$ GPC3-CAR-T cells demonstrated higher anti-tumor activity against PD-L1-expressing HCC cell PLC/ $\mathrm{PRF} / 5$ than WT CAR-T cells in a CAR-dependent manner. Moreover, PD-1 deficiency did not affect T cell subsets and activation status of CAR-T cells. Notably, co-culture of PD- $1^{-/}$GPC3-CAR-T cells with native PD-L1-expressing HCC did not induce CAR-T cell exhaustion. Furthermore, the knockout of PD-1 led to enhanced anti-tumor activity of CAR-T cells against HCC in vivo, and improved persistence and infiltration of CAR-T cells in tumor-bearing NOD-SCID IL-2receptor gamma null (NSG) mice. These findings suggest the improved anti-tumor capacity of PD-1 ${ }^{-1}$ CAR-T cells in HCC and the potential of precise gene editing on immune checkpoints to increase the efficacy of CAR-T cells ${ }^{[37]}$. In another study, Pan et al.$^{[38]}$ introduced a fusion protein composed of a PD-1 extracellular domain and $\mathrm{CH} 3$ from IgG4 into GPC3-specific CAR-T cells (GPC3-28Z). GPC3-CAR-T cells carrying the PD-1-CH3 fusion protein (sPD1) specifically recognized and lysed $\mathrm{GPC} 3^{+} \mathrm{HCC}$ cells, while secreting soluble PD-1 to impair PD-1/PDL1 signaling. The incorporation of soluble PD1 protected CAR-T cells from exhaustion when combating target cells. More importantly, GPC3-28Z-sPD1 T cells suppressed tumor xenograft growth significantly when compared with GPC3-28Z T cells in two HCC tumor xenograft models. The treatment of mice with GPC3-28Z-sPD1 T cells resulted in a higher number of $\mathrm{CD}^{+}{ }^{+} \mathrm{T}$ cells in the circulation as well as in tumors, enhanced granzyme B expression and reduced Ki67 expression in tumors. These findings suggest that GPC3-targeted CAR-T cells carrying soluble PD-1 hold great potential for the treatment of HCC.

The combination of GPC3-CAR-T cells and targeted therapy (angiogenesis inhibitor) in the treatment of HCC has been investigated. In an immunocompetent mouse model, mouse GPC3-CAR (mCAR) T cells showed potent growth suppressive activity against small tumors, but did not show suppressive activity against large, established tumors. Sorafenib (angiogenesis inhibitor), at a subpharmacologic but not a pharmacologic dose, enhanced the anti-tumor activity of mCAR-T cells, partially by increasing IL- 12 expression by TAMs and cancer cell apoptosis. Sorafenib, at both subpharmacologic and pharmacologic doses, had a weak effect on the function of human CAR (huCAR) T cells in an immunodeficient mouse model. However, huCAR-T cells and sorafenib together showed synergistic activities against tumor cells in vivo. Collectively, these findings suggest the potential of combining sorafenib with GPC3-targeted CAR-T cells in the treatment of HCC ${ }^{[39]}$.

Other than GPC3, CD133 is another promising therapeutic target for CAR-T cells, for it is expressed by stem cells of different cancer types. CD133-targeted CAR-T cells were prepared and its anti-tumor activity and toxicity were tested in a phase I clinical trial. In total, 23 patients with advanced and CD $133^{+}$tumors (14 HCC, 7 pancreatic carcinomas, and 2 colorectal carcinomas) were enrolled and received CD133-CAR-T cell infusion. Of 23 patients, 3 achieved partial remission, and 14 achieved stable disease. Repeat CAR-T cell infusions might provide a longer period of disease stability, especially in patients who have achieved tumor regression after the first ACT treatment. Of note, $91.3 \%$ of patients had not developed detectable de novo lesions during treatment. $\mathrm{CD}_{133^{+}}$cells were eliminated after CD133-CAR-T cell infusion and toxicity is well tolerated. This trial suggests that the infusion of CA133-CAR-T cells may be of value in treating $\mathrm{CD}_{133^{+}}$advanced cancers ${ }^{[40]}$.

NKG2D-NK group 2 member D (NKG2D) ligands (NKG2DL) are not expressed on the surface of normal cells but are overexpressed on malignant cells, thereby providing targets for CAR-T therapy. The expression levels of most NKG2DLs are higher in tumors than that in normal tissues. Due to this reason, NKG2D- 
based CAR-T cells were designed and prepared. NKG2D-CAR-T cells had an efficient killing effect in vitro on HCC cell lines SMMC-7721 and MHCC97H, which express high levels of NKG2DLs. However, they were less efficient in eradicating NKG2DL-silenced SMMC-7721 cells or NKG2DL-negative Hep3B cells. The overexpression of NKG2DL (MICA or ULBP2) in Hep3B resulted in enhanced killing capacity of NKG2D-CAR-T cells. Importantly, T cells expressing the NKG2D-BBz CAR effectively killed SMMC-7721 HCC xenografts. Taken together, these findings imply that NKG2D-CAR-T cells might hold great promise for future therapeutic intervention of NKG2DL-positive $\mathrm{HCC}^{[41]}$.

Zhang et al. ${ }^{[42]}$ prepared CD147-CAR-T cells that were inducible. Since CD147 is expressed at a low level on non-tumor cells, the Tet-On $3 \mathrm{G}$ system was introduced to induce CD147CAR expression to minimize the on-target, off-tumor toxicity. Specifically, Tet-On-CD147-CAR lentiviral plasmid (LV-Tet-CD147CAR) was constructed, of which CD147-CAR was controlled by the Tet-On 3G system. The presence of doxycycline (Dox) resulted in enhanced proliferation, cytotoxicity, and cytokine secretion of Tet-CD147-CAR-T cells. Notably, (Dox ${ }^{+}$) Tet-CD147-CAR-T cells significantly suppressed tumor growth in nude mice through multiple intra-tumoral administrations. Collectively, these findings suggest that CD147-CAR expression and activity were inducible, which reduced the toxicity of CAR-T cell therapy. Furthermore, the study offered evidence to support the potential benefits and translation of CD147-CAR-T cells for the treatment of HCC patients.

\section{Cytokine-induced killer}

Adjuvant cytokine-induced killer (CIK) cell-based immunotherapy is a promising therapeutic approach that increases overall survival and decreases relapses in HCC patients. Yoon et al ${ }^{[43]}$ performed a trial to investigate the efficacy of adjuvant immunotherapy with activated CIK cells for treating HCC patients. 59 patients with stage I or II HCC who had undergone surgery or radiofrequency ablation, followed by adjuvant CIK cell immunotherapy at two large-volume centers in Korea were paired with 59 matched control subjects. They found that adjuvant immunotherapy with autologous CIK cells after curative treatment prolonged relapse free survival (RFS) of patients with HCC. In a follow-up study of 226 patients with 114 in the immunotherapy group (treated with $6.4 \times 10^{9}$ CIK cells) and 112 controls (no treatment) after potentially curative treatment for HCC, the immunotherapy group was observed to have a significantly lower risk of relapse or death. The RFS rate was $44.8 \%$ in the immunotherapy group compared to $33.1 \%$ in the control group at 5 years. The risk of all-cause death was also decreased in the immunotherapy group $v s$. the control group. Furthermore, the significant increase in RFS and OS in the immunotherapy group lasted over 5 years ${ }^{[44]}$.

Yu et al. ${ }^{[45]}$ performed a systematic review and meta-analysis of published studies in order to evaluate the safety and efficacy of CIK cell-based immunotherapy as adjuvant therapy. This included eight randomized controlled trials (RCTs), six prospective studies, and three retrospective studies. The overall analysis indicated that CIK cell therapy resulted in an increased survival rate. CIK cells in non-RCTs resulted in improved progression-free survival, but in RCTs, had similar progression-free survival rates as those of controls. CIK cell-treatment also led to lower rates of relapse in RCTs and similar results were observed when non-RCTs and RCTs were combined. Collectively, these findings suggest that adjuvant CIK cell-based immunotherapy can enhance OS and decrease relapses in HCC patients.

PD-L1 expression may imply the presence of endogenous host immune responses to tumor. In one retrospective study including 448 HCC patients, 217 cases underwent hepatectomy and 231 were treated with both hepatectomy and post-operative CIK cell transfusion. CIK treatment led to a significantly improved prognosis compared to surgery alone. Higher expression levels of PD-L1 were observed in patients with long-term survival benefit in the CIK treatment group. Patients with more than 5\% PD-L1 expression also had better OS and RFS than those with $<5 \%$ PD-L1 expression, particularly in the 
subgroup with high hepatitis B viral load. PD-L1 expression however, did not correlate with patient survival in the surgery alone group ${ }^{[46]}$. These findings suggest that PD-L1 expression can serve as a biomarker for predicting survival benefit from adjuvant CIK cell immunotherapy in HCC patients.

\section{T cell receptor gene-engineered $\mathrm{T}$ cells}

Genetic engineering of TAA-specific T cell receptor (TCR) could be an option to yield AFP-specific CTLs. AFP is overexpressed in HCC and serves both as a TAA as well as a potential target for adoptive immunotherapy. However, the low percentage and exhausted AFP-specific T cells in vivo impedes adoptive immunotherapy. Genetic modification with TCRs that are specific for HCC-associated antigens, such as AFP, strongly redirect human T cells to specifically eliminate HCC tumor cells. Using lentivector and peptide immunization, Zhu et al. ${ }^{[47]}$ identified a population of $\mathrm{CD}^{+}{ }^{+} \mathrm{T}$ cells in HLA-A2 transgenic AAD mice that recognized the AFP158 epitope on human HCC cells. Adoptive injection of the AFP158-specific mouse $\mathrm{CD}^{+}{ }^{+} \mathrm{T}$ cells killed HepG 2 tumor xenografts in immunocompromised NSG mice. T cell hybridoma clones from the AFP158-specific mouse $\mathrm{CD}^{+}{ }^{+} \mathrm{T}$ cells were then established and three sets of paired TCR genes were identified out of five hybridomas. The murine TCR-expressing primary human $\mathrm{T}$ cells can bind to HLA-A2/AFP158 tetramer. TCR gene-engineered T (TCR-T) cells also specifically recognized and eliminated HLA-A2 ${ }^{+} \mathrm{AFP}^{+} \mathrm{HepG} 2 \mathrm{HCC}$ tumor cells, but had no toxic effect on normal primary hepatocytes in vitro. Notably, AFP-specific TCR-T cells could kill HepG2 tumors in NSG mice. These findings suggest that AFP158-specific TCRs have the ability to engineer HLA-A2-positive autologous T cells to treat patients with $\mathrm{HCC}^{[47]}$. Sun et al. ${ }^{[48]}$ used AFP158-166 peptide-loaded autologous DCs to stimulate AFPspecific CTLs. TCR genes of AFP-specific CTLs were then cloned into a lentiviral vector, which in turn infected nonspecific activated $\mathrm{T}$ cells. The specific cytotoxic activity against HpeG2 in vitro and in tumorbearing NOD/SCID mice was significantly enhanced in engineered CTLs than that in AFP-specific CTLs stimulated by peptide-loaded DCs or controls. TCR gene transfer is thus a promising strategy to yield AFPspecific CTLs for HCC ${ }^{[48]}$.

In China, around $85 \%$ of HCC is associated with HBV infection. Cells from most HBV-associated HCCs contain HBV-DNA fragments that fail to encode entire HBV antigens, but do encode epitopes of HBVspecific T cells. Given that, autologous TCR-T cells that recognize epitopes from HBV-DNA in patients' metastases were infused into two patients without notable toxicity. This strategy might be exploited for a wider population of patients with HBV-related $\mathrm{HCC}^{[49]}$. Qasim et al ${ }^{[50]}$, also showed HBV antigen expression in HCC metastases. T cells were then genetically engineered to express an HBsAg specific TCR to treat chemo-resistant extrahepatic metastases. Genetically-engineered T cells reduced HBsAg levels without exacerbation of liver inflammation or other adverse effects. This study further supports this approach in treating patients with HBV-associated $\mathrm{HCC}^{[50]}$.

\section{Gamma-delta T cells}

Gamma-delta $(\gamma \delta)$ T cells have been found to be promising as cellular immunotherapy in HCC patients ${ }^{[51]}$. The amplifying ability of circulating $\gamma \delta \mathrm{T}$ cells was related with the clinicopathological characteristics of patients, such as clinical stage, levels of AFP and albumin, duration of disease, size and number of tumors, numbers of Tregs and $\gamma \delta \mathrm{T} 17$ cells, and levels of IL-17A. Notably, the frequency of $\gamma \delta \mathrm{T}$ cells that were positive for IFN- $\gamma$, TNF- $\alpha$, granzyme B, perforin, and lysosome-associated membrane protein remained unchanged both before and after amplification. More importantly, the in vitro cytotoxicity of $\gamma \delta \mathrm{T}$ cells remained unchanged, which may render them feasible for HCC immunotherapy. However, adoptive transfer of $\gamma \delta$ T cells should be individualized based on the clinicopathological features of patients ${ }^{[51]}$.

\section{Natural killer cells}

Natural killer (NK) cells play a pivotal role in eradicating virus-infected and transformed cells in the innate immune system. Kamiya et al. ${ }^{[52]}$ successfully expanded NK cells from peripheral blood of healthy donors by using the K562-mb15-41BBL cell line as a stimulus. Following expansion, NK cells showed great 


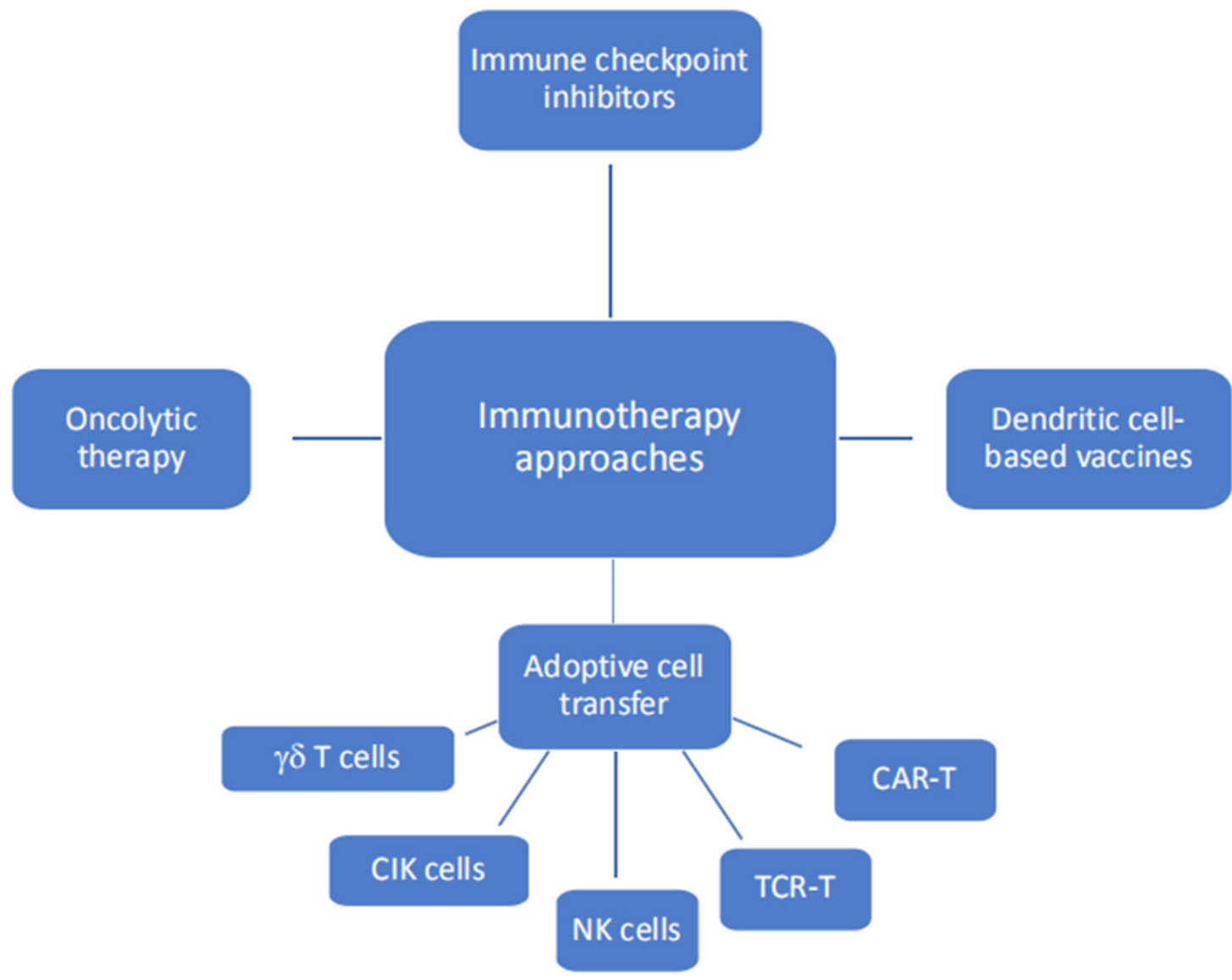

Figure 1. An overview of the different immunotherapy approaches for the treatment of hepatocellular carcinoma, which includes immune checkpoint inhibitor antibodies, adoptive cell transfer, dendritic cell-based vaccines and oncolytic therapy. CIK: cytokineinduced killer; NK: natural killer; TCR: T cell receptor; CAR: chimeric antigen receptor

cytotoxic activity against HCC cell lines in vitro. In immunodeficient NOD/scid IL2RG null mice engrafted with Hep3B, adoptive transfer of expanded NK cells resulted in markedly decreased tumor growth and enhanced overall survival. Expanded NK cells can further enhance the sorafenib-mediated anti-tumor effect on HCC cells. In addition, CAR-NK cells (NKG2D-CD3zeta-DAP10) were prepared and found to have increased cytotoxicity in vitro and in immunodeficient mice. CAR-NK cells have now been adapted for clinical-grade conditions ${ }^{[52]}$.

In one retrospective study, autologous mononuclear cells were induced into NK cells, $\gamma \delta \mathrm{T}$ cells, and CIK cells and then infused intravenously to HCV-associated HCC patients (study group treated with a combination of ACT and conventional therapy). Compared to controls (conventional therapy), the study group showed improved prognosis and decreased virus load in HCV-related HCC patients, without impairment of liver function ${ }^{[33]}$.

\section{ADVANCEMENT OF ONCOLYTIC IMMUNOTHERAPY}

Oncolytic immunotherapy is another strategy for cancer treatment which utilizes live or heat-inactivated viruses, replicating within and destroying tumor cells. Pexastimogene devacirepvec (Pexa-Vec) is a vaccinia virus-based oncolytic immunotherapy that can destroy tumor cells and enhance anti-tumor immunity by expressing GM-CSF. A phase IIa trial in predominantly sorafenib-naïve HCC showed an OS benefit. In order to investigate whether the combination of Pexa-Vec with best supportive care (BSC) could increase OS compared to BSC alone in HCC patients who did not respond to sorafenib therapy, 129 patients were 
recruited and divided into the experimental (combination of Pexa-Vec with BSC) and the control (BSC alone) groups. Although Pexa-Vec was generally well-tolerated and could induce immune responses to vaccinia and HCC associated antigens, it failed to enhance OS as a second-line treatment after sorafenib failure. This class of oncolytic immunotherapy may benefit cancer patients with earlier disease stages and the combination of this approach with other therapeutics needs to be investigated ${ }^{[54]}$.

NCT03071094 is another trial to evaluate the safety and efficacy of Pexa-Vec plus PD-1 inhibitors as firstline therapy for advanced HCC. However, no results have been released.

\section{CONCLUSION}

We have summarized the immunotherapy approaches for the treatment of HCC patients [Figure 1]. Increasing data provide evidence that HCC patients can benefit from different immunotherapies. Currently, only anti-PD-1/PD-L1 antibodies have been approved to treat advanced HCC as a first-line therapy, while other immunotherapy approaches are still in clinical trials. However, given that only a proportion of HCC patients respond to anti-PD-1/PD-L1 antibodies, combination therapy will likely be the future. Final results of ongoing trials are crucial for the design and development of further combination therapy in HCC according to its efficacy and safety profile. In addition, future studies should explore biomarkers to predict one's response to immunotherapy in HCC, other than PD-L1 expression in terms of ICB therapy, novel target antigens (neo-antigens) to prepare DC-based vaccines and CAR-T cells. Future clinical trials are necessary. Indeed, we have opened the doors to immunotherapy and complete control of HCC may not a dream.

\section{DECLARATIONS}

\section{Authors' contributions}

Wrote the review: Ni L, Dong C

Helped with review writing: Feng Y

\section{Availability of data and materials}

Not applicable.

\section{Financial support and sponsorship}

This work was supported by grants from Beijing Municipal Science and Technology (Grant No. Z181100001318007) and National Natural Science Foundation of China (NSFC) (Grant No. 31991173).

\section{Conflicts of interest}

All authors declared that there are no conflicts of interest.

\section{Ethical approval and consent to participate}

Not applicable.

\section{Consent for publication}

Not applicable.

\section{Copyright}

(c) The Author(s) 2020.

\section{REFERENCE}

1. Zhu RX, Seto WK, Lai CL, Yuen MF. Epidemiology of hepatocellular carcinoma in the Asia-Pacific Region. Gut Liver 2016;10:332-9.

2. Yang JD, Roberts LR. Hepatocellular carcinoma: a global view. Nat Rev Gastroenterol Hepatol 2010;7:448-58. 
3. Llovet JM, Ricci S, Mazzaferro V, Hilgard P, Gane E, et al. Sorafenib in advanced hepatocellular carcinoma. N Engl J Med 2008;359:378-90.

4. Buonaguro L, Mauriello A, Cavalluzzo B, Petrizzo A, Tagliamonte M. Immunotherapy in hepatocellular carcinoma. Ann Hepatol 2019;18:291-7.

5. Brown ZJ, Greten TF, Heinrich B. Adjuvant treatment of hepatocellular carcinoma: prospect of immunotherapy. Hepatology 2019;70:1437-42.

6. Obeid JM, Kunk PR, Zaydfudim VM, Bullock TN, Slingluff CL, Jr., Rahma OE. Immunotherapy for hepatocellular carcinoma patients: is it ready for prime time? Cancer Immunol Immunother 2018;67:161-74.

7. El-Khoueiry AB, Sangro B, Yau T, Crocenzi TS, Kudo M, et al. Nivolumab in patients with advanced hepatocellular carcinoma (CheckMate 040): an open-label, non-comparative, phase 1/2 dose escalation and expansion trial. Lancet 2017;389:2492-502.

8. Leonardi GC, Candido S, Cervello M, Nicolosi D, Raiti F, et al. The tumor microenvironment in hepatocellular carcinoma (review). Int J Oncol 2012;40:1733-47.

9. Zhang Q, He Y, Luo N, Patel SJ, Han Y, et al. Landscape and dynamics of single immune cells in hepatocellular carcinoma. Cell 2019;179:829-45.e20.

10. Chew V, Lai L, Pan L, Lim CJ, Li J, et al. Delineation of an immunosuppressive gradient in hepatocellular carcinoma using highdimensional proteomic and transcriptomic analyses. Proc Natl Acad Sci U S A 2017;114:E5900-9.

11. Zhang Z, Ma L, Goswami S, Ma J, Zheng B, et al. Landscape of infiltrating B cells and their clinical significance in human hepatocellular carcinoma. Oncoimmunology 2019;8:e1571388.

12. Ye L, Zhang Q, Cheng Y, Chen X, Wang G, et al. Tumor-derived exosomal HMGB1 fosters hepatocellular carcinoma immune evasion by promoting TIM-1(+) regulatory B cell expansion. J Immunother Cancer 2018;6:145.

13. Xue H, Lin F, Tan H, Zhu ZQ, Zhang ZY, et al. Overrepresentation of IL-10-expressing B cells suppresses cytotoxic CD4+ T cell activity in HBV-induced hepatocellular carcinoma. PLoS One 2016;11:e0154815.

14. Yarchoan M, Hopkins A, Jaffee EM. Tumor mutational burden and response rate to PD-1 inhibition. N Engl J Med 2017;377:2500-1.

15. Ou DL, Lin YY, Hsu CL, Lin YY, Chen CW, et al. Development of a PD-L1-expressing orthotopic liver cancer model: implications for immunotherapy for hepatocellular carcinoma. Liver Cancer 2019;8:155-71.

16. Zheng Y, Wang T, Tu X, Huang Y, Zhang H, et al. Gut microbiome affects the response to anti-PD-1 immunotherapy in patients with hepatocellular carcinoma. J Immunother Cancer 2019;7:193.

17. Kaseb AO, Vence L, Blando J, Yadav SS, Ikoma N, et al. Immunologic correlates of pathologic complete response to preoperative immunotherapy in hepatocellular carcinoma. Cancer Immunol Res 2019;7:1390-5.

18. Hong YK, Li Y, Pandit H, Li S, Pulliam Z, et al. Epigenetic modulation enhances immunotherapy for hepatocellular carcinoma. Cell Immunol 2019;336:66-74.

19. Zhan C, Ruohoniemi D, Shanbhogue KP, Wei J, Welling TH, et al. Safety of combined yttrium-90 radioembolization and immune checkpoint inhibitor immunotherapy for hepatocellular carcinoma. J Vase Interv Radiol 2020;31:25-34.

20. Wesley JD, Whitmore J, Trager J, Sheikh N. An overview of sipuleucel-T: autologous cellular immunotherapy for prostate cancer. Hum Vaccin Immunother 2012;8:520-7.

21. Gao F, Zhu HK, Zhu YB, Shan QN, Ling Q, et al. Predictive value of tumor markers in patients with recurrent hepatocellular carcinoma in different vascular invasion pattern. Hepatobiliary Pancreat Dis Int 2016;15:371-7.

22. Shimizu Y, Suzuki T, Yoshikawa T, Endo I, Nakatsura T. Next-generation cancer immunotherapy targeting glypican-3. Front Oncol 2019;9:248.

23. Sang M, Wang L, Ding C, Zhou X, Wang B, et al. Melanoma-associated antigen genes - an update. Cancer Lett 2011;302:85-90.

24. Castelli C, Rivoltini L, Andreola G, Carrabba M, Renkvist N, et al. T-cell recognition of melanoma-associated antigens. J Cell Physiol 2000;182:323-31.

25. Wang K, Liu J, Yan ZL, Li J, Shi LH, et al. Overexpression of aspartyl-(asparaginyl)-beta-hydroxylase in hepatocellular carcinoma is associated with worse surgical outcome. Hepatology 2010;52:164-73.

26. Lee JH, Lee Y, Lee M, Heo MK, Song JS, et al. A phase I/IIa study of adjuvant immunotherapy with tumour antigen-pulsed dendritic cells in patients with hepatocellular carcinoma. Br J Cancer 2015;113:1666-76.

27. Lee JH, Tak WY, Lee Y, Heo MK, Song JS, et al. Adjuvant immunotherapy with autologous dendritic cells for hepatocellular carcinoma, randomized phase II study. Oncoimmunology 2017;6:e1328335.

28. Zhou Y, Liu F, Li C, Shi G, Xu X, et al. Construction and characterization of adenovirus vectors encoding aspartate-beta-hydroxylase to preliminary application in immunotherapy of hepatocellular carcinoma. J Immunol Res 2018;2018:9832467.

29. Chen C, Ma YH, Zhang YT, Zhang F, Zhou N, et al. Effect of dendritic cell-based immunotherapy on hepatocellular carcinoma: a systematic review and meta-analysis. Cytotherapy 2018;20:975-89.

30. Mo HY, Liao YY, You XM, Cucchetti A, Yuan BH, et al. Timely meta-analysis on the efficacy of adoptive immunotherapy for hepatocellular carcinoma patients after curative therapy. PLoS One 2017;12:e174222.

31. Ding M, Wang Y, Chi J, Wang T, Tang X, et al. Is adjuvant cellular immunotherapy essential after TACE-predominant minimally-invasive treatment for hepatocellular carcinoma? A systematic meta-analysis of studies including 1774 patients. PLoS One 2016;11:e0168798.

32. Liu Y, Chen X, Han W, Zhang Y. Tisagenlecleucel, an approved anti-CD19 chimeric antigen receptor T-cell therapy for the treatment of leukemia. Drugs Today (Barc) 2017;53:597-608.

33. Gao H, Li K, Tu H, Pan X, Jiang H, Shi B, et al. Development of T cells redirected to glypican-3 for the treatment of hepatocellular carcinoma. Clin Cancer Res 2014;20:6418-28.

34. Jiang Z, Jiang X, Chen S, Lai Y, Wei X, et al. Anti-GPC3-CAR T cells suppress the growth of tumor cells in patient-derived xenografts of 
hepatocellular carcinoma. Front Immunol 2016;7:690.

35. Wang P, Qin W, Liu T, Jiang D, Cui L, et al. PiggyBac-engineered T cells expressing a glypican-3-specific chimeric antigen receptor show potent activities against hepatocellular carcinoma. Immunobiology 2020;225:151850.

36. Chen C, Li K, Jiang H, Song F, Gao H, et al. Development of T cells carrying two complementary chimeric antigen receptors against glypican-3 and asialoglycoprotein receptor 1 for the treatment of hepatocellular carcinoma. Cancer Immunol Immunother 2017;66:475-89.

37. Guo X, Jiang H, Shi B, Zhou M, Zhang H, et al. Disruption of PD-1 enhanced the anti-tumor activity of chimeric antigen receptor T cells against hepatocellular carcinoma. Front Pharmacol 2018;9:1118.

38. Pan Z, Di S, Shi B, Jiang H, Shi Z, et al. Increased antitumor activities of glypican-3-specific chimeric antigen receptor-modified T cells by coexpression of a soluble PD1-CH3 fusion protein. Cancer Immunol Immunother 2018;67:1621-34.

39. Wu X, Luo H, Shi B, Di S, Sun R, et al. Combined antitumor effects of Sorafenib and GPC3-CAR T cells in mouse models of hepatocellular carcinoma. Mol Ther 2019;27:1483-94.

40. Wang Y, Chen M, Wu Z, Tong C, Dai H, et al. CD133-directed CAR T cells for advanced metastasis malignancies: a phase I trial. Oncoimmunology 2018;7:e1440169.

41. Sun B, Yang D, Dai H, Liu X, Jia R, et al. Eradication of hepatocellular carcinoma by NKG2D-based CAR-T cells. Cancer Immunol Res 2019;7:1813-23.

42. Zhang RY, Wei D, Liu ZK, Yong YL, Wei W, et al. Doxycycline inducible chimeric antigen receptor T cells targeting CD147 for hepatocellular carcinoma therapy. Front Cell Dev Biol 2019;7:233.

43. Yoon JS, Song BG, Lee JH, Lee HY, Kim SW, et al. Adjuvant cytokine-induced killer cell immunotherapy for hepatocellular carcinoma: a propensity score-matched analysis of real-world data. BMC Cancer 2019;19:523.

44. Wang L, Simons DL, Lu X, Tu TY, Solomon S, et al. Connecting blood and intratumoral Treg cell activity in predicting future relapse in breast cancer. Nat Immunol 2019;20:1220-30.

45. Yu R, Yang B, Chi X, Cai L, Liu C, et al. Efficacy of cytokine-induced killer cell infusion as an adjuvant immunotherapy for hepatocellular carcinoma: a systematic review and meta-analysis. Drug Des Devel Ther 2017;11:851-64.

46. Chen CL, Pan QZ, Zhao JJ, Wang Y, Li YQ, et al. PD-L1 expression as a predictive biomarker for cytokine-induced killer cell immunotherapy in patients with hepatocellular carcinoma. Oncoimmunology 2016;5:e1176653.

47. Zhu W, Peng Y, Wang L, Hong Y, Jiang X, et al. Identification of alpha-fetoprotein-specific T-cell receptors for hepatocellular carcinoma immunotherapy. Hepatology 2018;68:574-89.

48. Sun L, Guo H, Jiang R, Lu L, Liu T, et al. Engineered cytotoxic T lymphocytes with AFP-specific TCR gene for adoptive immunotherapy in hepatocellular carcinoma. Tumour Biol 2016;37:799-806.

49. Tan AT, Yang N, Lee Krishnamoorthy T, Oei V, Chua A, et al. Use of expression profiles of HBV-DNA integrated into genomes of hepatocellular carcinoma cells to select T cells for immunotherapy. Gastroenterology 2019;156:1862-76.e9.

50. Qasim W, Brunetto M, Gehring AJ, Xue SA, Schurich A, et al. Immunotherapy of HCC metastases with autologous T cell receptor redirected T cells, targeting HBsAg in a liver transplant patient. J Hepatol 2015;62:486-91.

51. Tian W, Ma J, Shi R, Ren C, He J, et al. gammadelta T cell-mediated individualized immunotherapy for hepatocellular carcinoma considering clinicopathological characteristics and immunosuppressive factors. Oncol Lett 2018;15:5433-42.

52. Kamiya T, Chang YH, Campana D. Expanded and activated natural killer cells for immunotherapy of hepatocellular carcinoma. Cancer Immunol Res 2016;4:574-81.

53. Qian L, Wang N, Tian H, Jin H, Zhao H, et al. Dual Effects of cellular immunotherapy in inhibition of virus replication and prolongation of survival in HCV-positive hepatocellular carcinoma patients. J Immunol Res 2016;2016:6837241.

54. Moehler M, Heo J, Lee HC, Tak WY, Chao Y, et al. Vaccinia-based oncolytic immunotherapy Pexastimogene Devacirepvec in patients with advanced hepatocellular carcinoma after sorafenib failure: a randomized multicenter Phase IIb trial (TRAVERSE). Oncoimmunology 2019;8:1615817. 